\title{
Definiendo a los otros: relatos académicos sobre la diversidad en la escuela
}

\author{
Defining the others: academic narratives about diversity at school
}

\author{
María Luisa Jiménez-Rodrigo / mljimenez@us.es \\ Universidad de Sevilla, España \\ Raquel Guzmán-Ordaz / r.guzman@usal.es \\ Universidad de Salamanca, España
}

\begin{abstract}
The present paper reviews Spanish social scientific literature analyzing the employment of the "diversity" concept applied to the school field. The research methodology started with detailed scrutiny and examination of the Social Sciences journals indexed in CSIC-ISOC and DIALNET databases, published between 2006 and 2012, followed by the documentary analysis of 218 articles extracted from those sources. Discourse analysis enables us to single out distinct narrative configurations about how "the other" is defined at school grounds. Results show two hegemonic narrative patterns: an institutional narrative based upon the legal sorting of students classified by scholar results and abilities, and an intercultural narrative constructed around cultural categories that would differentiate nationals from foreigners. We conclude that both accounts unveil predominantly essentialized views of the "diversity" idea and its conceptualization, failing to ponder over other social and economic categories when addressing school related inequalities and differences.
\end{abstract}

Keywords: cultural diversity, literature analysis, social construction of reality, interculturality.

Resumen: Este artículo indaga en los usos académicos del concepto diversidad en el ámbito escolar a partir del análisis de la literatura científica española. El método se basa en el análisis documental partiendo de una exhaustiva búsqueda y revisión de 218 artículos publicados entre 2006 y 2012 en revistas indexadas en CSIC-ISOC y DIALNET. Se aplicó un esquema estructural de análisis que permitió identificar diferentes configuraciones narrativas en torno a la definición del "otro" en la escuela. Los resultados señalan dos relatos hegemónicos: institucional, basado en las clasificaciones legislativas del alumnado en función de sus capacidades y rendimientos escolares; e intercultural, sustentado en categorizaciones en torno a las diferencias culturales derivadas de la condición inmigrante-extranjera. Concluimos que en ambos relatos se proyecta predominantemente una visión esencializada de la diversidad construida desde parámetros dicotómicos y excluyentes, sin apenas considerar otras categorías sociales y económicas en la conformación de las diferencias y desigualdades educativas.

Palabras clave: diversidad cultural, análisis de la literatura, construcción social de la realidad, interculturalidad. 


\section{Introducción}

La cuestión de la diversidad ha adquirido una significativa relevancia en los debates sobre educación, cristalizándose dentro de algunos discursos pedagógicos, políticos y académicos como una omnipresente y escasamente cuestionada categoría de pensamiento, interpretación y ordenación de la realidad educativa. En España, esta preocupación por la diversidad en las aulas ha dado lugar en las últimas décadas a una gran profusión, sobre todo amparada e impulsada por las reformas educativas implantadas desde 1990 y los debates sobre la multiculturalidad, de estudios, planes y propuestas de enseñanza y aprendizaje que utilizan esta noción como eje central de reflexión e intervención escolar para la inclusión de colectivos de estudiantes identificados como diferentes (Coll, 2002; Monsalvo y Carbonero, 2005).

En este sentido, se homologa lo diverso a lo diferente y se expande una red tejida de los distintos tipos de diferencias que pueden identificarse en la escuela: diferencias de habilidades, de capacidades de aprendizaje y de orígenes étnicos y nacionales (Gimeno, 2000). Nos encontramos, pues, frente a un concepto que se ha erigido como una exitosa fórmula de estudio [buzzword] en el ámbito académico y escolar, pero cuyos sentidos no están suficientemente determinados ni esclarecidos.

De hecho, el término diversidad ha sido calificado como "palabra blanda", "eufemismo", "tópico" o "retórica de moda”, reprochándose su uso automático sin la suficiente reflexión teórica y precisión conceptual (Colectivo IOE, 1997; Duschatzky y Skliar, 2001; Pérez de Lara, 2001; Terrén, 2001; Skliar, 2005). El empleo de la diversidad como categoría descriptiva y analítica de la realidad escolar se caracteriza, además, por su creciente polisemia al aplicarse a situaciones y ámbitos tan variados como los vinculados con la edad, el género, la religión o la orientación sexual (Zapata, 2008-2009), lo cual ha derivado en múltiples e, incluso, contradictorias acepciones (Almeida et al., 2010; Marvasti y McKinney, 2011; Ramos, 2012).

La utilización del concepto de diversidad, pese a que es habitualmente presentado como una representación o reflejo de un hecho tan aparentemente "obvio" y "tan natural como la vida misma" (Gimeno, 2000; Ramos, 2012), no es neutral ni socialmente aséptica. Como la sociología constructivista ha evidenciado, todo concepto que se aplica para pensar e interpretar determinada realidad social también contribuye a su identificación y caracterización, señalando qué aspectos, sujetos o problemas son merecedores de atención y cómo éstos son socialmente caracterizados y valorados (Berger y Luckmman, 2006). 
En el caso de la diversidad, estas cuestiones son especialmente relevantes porque su empleo arrastra efectos clasificadores y catalogadores de determinados sujetos y grupos a partir de la tipificación de ciertas diferencias (Duschatzky y Skliar, 2001). De esta forma, el uso de la diversidad implicaría "un proceso de representación, una construcción simbólica de un 'otro' y por lo tanto de un 'nosotros' en relación al cual el otro es entendido como diferente o como diverso" (Almeida et al., 2010: 33).

Este "proceso de diferencialismo" inscribe a sujetos específicos en una alteridad que los nombra y los construye como los depositarios de unas marcas que los hacen ser "diferentes" (Skliar, 2005). Este proceso implica la definición de dispositivos clasificatorios que, lejos de ser arbitrarios, responden a una selección y jerarquización de determinadas cualidades y características sobre otras, constituyéndose en una operación de exclusión respecto a la norma o lo normalizado (Almeida et al., 2010).

Es preciso que alguien señale quiénes son los diferentes y en qué sentido lo son. Estas operaciones de señalamiento y delimitación de la alteridad tienen lugar dentro de relaciones asimétricas y de poder, donde determinados agentes -situados en un polo dominante- tienen y ejercen su poder para identificar, designar y describir a otros sujetos, ubicados habitualmente en el polo dominado (Terrén, 2001).

Por las implicaciones que los procesos de categorización y etiquetaje social generan en la estructuración de las relaciones sociales entre grupos, resulta de utilidad atender y entender el impacto que los dispositivos clasificatorios que los discursos hegemónicos sobre la diversidad promueven. Los discursos, en tanto construcciones sociales, configuran la realidad y revisten importancia para los agentes implicados en los procesos de creación y legitimación de la misma. Pero además, el análisis sobre las configuraciones narrativas (Conde, 2009) puede operar como estructura analítica para detectar los sesgos discursivos factibles de ser evitados en el ámbito de los policy makers, e incluso para las prácticas inclusivas de los propios actores escolares.

Toda esta problemática y controversia que envuelve al empleo de la diversidad como categoría analítica y de política educativa ha llevado al planteamiento de este trabajo, ${ }^{1}$ cuyo objetivo es analizar, a partir del examen de la literatura

1 Trabajo realizado dentro del proyecto $\mathrm{I}+\mathrm{D}+\mathrm{i}$ "Escuela, comunidad e interculturalidad: estudio de los procesos inter-culturales e inter-actorales ante la gestión de la diversidad cultural en los centros educativos" (EDU2010-15808), financiado por el Ministerio de Ciencia e Innovación del Gobierno de España, y del proyecto de excelencia de la Junta de Andalucía "Estrategias innovadoras en Educación Intercultural: estudio de las distintas gramáticas de la gestión de la diversidad en los centros educativos" (SEJ-6329). 
académica española, los usos que se hacen de este concepto dentro de la investigación e intervención en el ámbito escolar, así como sus efectos en los procesos discursivos de construcción de la diferencia.

El presente estudio se sitúa dentro de una tradición internacional de investigaciones y debates sobre la construcción discursiva de la categoría diversidad. Estos estudios se han desarrollado fundamentalmente en el contexto estadounidense en torno a la cuestión de la raza y la categorización de los grupos escolares respecto la normatividad "blanca" y se han centrado en el análisis del discurso expresado por docentes y estudiantes universitarios (Ahmed, 2007; Berrey, 2011; Marvasti y McKinney, 2011; Bhopal y Rhamie, 2014) y en el discurso educativo institucional (VanDeventer, 2007).

En el contexto europeo y español, los estudios disponibles se fijan sobre todo en el análisis de los discursos del profesorado (Colectivo IOE, 1997; Terrén, 2001; Coronel y Gómez-Hurtado, 2015; Carrasco, 2015) en torno a la diferencia cultural, definida principalmente sobre la base del origen nacional, o a las necesidades educativas específicas (Lawson, Boyask y Waite, 2013).

Este estudio, frente a otros focalizados en los discursos docentes, representa una aproximación distinta y complementaria al ocuparse de los discursos académicos. Nos detenemos en la producción discursiva de los agentes académicos por su papel central y hegemónico en la construcción y legitimación de los discursos sobre la escuela. A este respecto, los agentes académicos, como "fabricantes de conocimiento", controlan legítimamente la producción y la articulación de los discursos dominantes (Van Dijk, 2009: 65-66) y logran definir el "saber oficial" (Berger y Luckmann, 2006: 158). Dirigimos, por tanto, nuestra mirada "hacia arriba" (Nader, 1972), hacia las élites simbólicas y su papel en el establecimiento de los mecanismos de definición de nuevos sujetos diferenciales, mediante la institucionalización de conceptos y categorías.

Como apunta Eduardo Menéndez (2002: 251), "los conceptos no deberían ser considerados como cristalizaciones originales, cuya 'pureza' hay que conservar, ya que al menos en parte serán inevitablemente modificados por quienes los usen teórica, empírica y/o prácticamente en función de sus objetivos, intereses y transacciones". Por ello, más que desvelar la formulación "original" del concepto, en este trabajo se describen y analizan los usos que se han venido realizando dentro de los discursos académicos recientes en torno a la diversidad en el ámbito de la escuela, a partir del examen de la literatura científica española. 
Así también, nos interesan los efectos sociales del concepto diversidad mediante la creación y definición barreras entre grupos, en línea de lo planteado por Clifford Geertz (1996: 87) respecto a la diversidad cultural:

Los usos de la diversidad cultural, de su estudio, su descripción, su análisis y su comprensión consisten menos en nuestras propias clasificaciones que nos separan de los demás y a los demás de nosotros por mor de defender la integridad del grupo y mantener la lealtad hacia él, que en definir el terreno que la razón debe atravesar si se quieren alcanzar y ver cumplidas sus modestas recompensas. Es éste un terreno desigual, lleno de repentinas fallas y pasos peligrosos donde los accidentes pueden suceder y suceden, y atravesarlo, o intentar hacerlo, poco o nada tiene que ver con allanarlo hasta hacer de él una llanura uniforme, segura y sin fisuras, sino que simplemente saca a la luz sus grietas y contornos.

Desde esta perspectiva, el presente estudio sobre los relatos académicos sobre la diversidad en el campo escolar incorpora el análisis de los efectos clasificatorios y definitorios de las diferencias, así como de las complejas, desiguales y conflictivas relaciones en torno al "nosotros" y a los "otros" que involucran los usos de la diversidad como categoría.

\section{Método}

La metodología empleada se sustenta en la revisión sistemática y análisis de la literatura académica publicada en las revistas científicas españolas en torno al concepto de diversidad aplicado al contexto de la escuela. Entre los múltiples productos de la labor académica, se seleccionaron como objeto de análisis los artículos científicos por su creciente relevancia y significación en la difusión, intercambio y construcción del saber experto.

El recorte analítico en torno a la producción científica indexada responde al interés de este trabajo por examinar los discursos hegemónicos en el campo académico, cuyos criterios formales de prestigio se articulan en torno a los índices de impacto de las publicaciones. ${ }^{2}$ Para la constitución del corpus documental se realizó una búsqueda exhaustiva de todos aquellos artículos publicados en revistas científicas españolas que incluyeran en su título o palabras clave el término "diversidad".

2 En un trabajo anterior (Jiménez-Rodrigo y Guzmán-Ordaz, 2013) se analizan las implicaciones de las reglas de juego del campo académico en la configuración de los discursos académicos sobre diversidad y que marcan las posibilidades de legitimación de las diferentes configuraciones narrativas. 
Para ello, se consultaron las dos principales bases de datos de referencia e indexación de la producción científica española: los sumarios de Ciencias Sociales y Humanidades (ISOC) del Consejo Superior de Investigaciones Científicas (CSIC) y el repositorio bibliográfico DIALNET de la Universidad de La Rioja.

El periodo de búsqueda se delimitó a los años que van desde 2006 -año de implantación de la Ley Orgánica de Educación (posteriormente, LOE) y que supuso un significativo hito en la institucionalización y normativización de la atención a la diversidad- hasta 2012. Junto a los temáticos, otros criterios de inclusión en el corpus documental residieron en que los artículos fueran trabajos de investigación, aportaciones teóricas o análisis/evaluación de intervenciones educativas, que éstos se refirieran a la realidad escolar española y que el artículo se encontrara disponible a texto completo.

Finalmente, se conformó un corpus de análisis de 218 artículos. Para contrarrestar los sesgos personales de selección del material, se replicó la búsqueda en varios momentos de la investigación y por distintas personas del equipo.

Los textos fueron analizados siguiendo un esquema estructural con el objeto de identificar el sentido de los artículos mediante la definición de regularidades de sus narraciones (Alonso y Fernández, 2006). Para ello, identificamos cuatro dimensiones de análisis (véase Cuadro $1^{3}$ ). La primera indaga en los argumentos utilizados para justificar la necesidad, relevancia y pertinencia de atender a la diversidad en el contexto de la escuela. La segunda hace referencia a los sentidos y usos de esta noción en relación con sus implicaciones clasificatorias y de diferenciación entre grupos y categorización de los sujetos "diferentes". La tercera alude a las valoraciones sobre el hecho de la diversidad y las atribuciones subjetivas que se plasman en los discursos académicos.

Estas valoraciones arrastran y legitiman determinadas respuestas que se demandan desde los textos a las instituciones educativas y políticas, junto a la propuesta de soluciones pedagógicas de cara a conseguir ciertas metas educativas y sociales. Estos últimos aspectos integran la cuarta dimensión de análisis.

En torno a dichas dimensiones, los artículos fueron, por una parte, codificados cuantitativamente a partir de sus características formales, perfiles de autoría y definiciones de la diversidad. Por otra parte, se analizó cualitativamente el discurso sobre las motivaciones y argumentos para tratar la diversidad en la escuela.

Esto permitió identificar las definiciones, sentidos y valoraciones en torno al concepto, así como las características utilizadas para su distinción junto a las respuestas planteadas frente al hecho de la diversidad. Se empleó el programa

3 Los cuadros 1 y 2 y el gráfico se encuentran en el Anexo, al final del presente texto (Nota del Editor). 
Atlas.ti 7 para organizar los textos y codificar su contenido. El proceso de codificación, al igual que el de selección de material, fue triangulado y contrastado entre diversas personas del equipo investigador. Esta estructura de análisis sirvió para identificar diferentes configuraciones narrativas (Conde, 2009: 168-169) que pudieran darse en torno a la diversidad con base en la exploración y polarización de las tensiones, los conflictos y las distintas posiciones contenidos en los discursos respecto a las dimensiones de análisis seleccionadas.

Conde (2009: 168) define las configuraciones narrativas como "una operación analítica que consiste en elegir y seleccionar aquellas dimensiones del texto que dando cuenta literal del mismo (...) permiten al mismo tiempo polarizarlo y ponerlo en relación tanto con el contexto social en el que se ha producido, como en los objetivos de la investigación". Las configuraciones narrativas han permitido, a su vez, desde el análisis sociológico, identificar la fortaleza simbólica del poder de los discursos, además de proveer de un marco de análisis y reflexión sobre cómo el contexto se expresa en el texto (Conde, 2009: 206).

Además, la consideración de las configuraciones narrativas permite ordenar la totalidad de los discursos en torno a la diversidad, así como el análisis interno de los textos que se producen respecto al concepto en el ámbito de la escuela en el contexto español.

\section{Resultados}

El análisis de los más de doscientos artículos recogidos revela la coexistencia de dos relatos hegemónicos, si bien éstos presentan variaciones o deslizamientos en su concepción de la diversidad (véase Gráfico 1). Por una parte, destaca el relato institucional, basado en las definiciones y clasificaciones del alumnado establecidas a priori por la legislación educativa española en función de las capacidades, trayectorias y rendimientos escolares.

El otro relato mayoritario es el intercultural, el cual se centra en la dimensión cultural de la diversidad, entendida predominantemente en relación con la condición inmigrante extranjera. Estos relatos dominantes aglutinan la mayoría (50,5\%: el institucional, y 40,4\%: el cultural) de los artículos examinados. Son dos relatos que el análisis de las configuraciones narrativas ha revelado como líneas discursivas distintas y distantes en cuanto a las dimensiones analíticas consideradas, y que se sintetizan en el Cuadro 2.

Este análisis contiene elementos de la construcción tipo-ideal weberiana, de forma que, para determinar estos relatos, se ha procedido, a partir del examen de los textos individuales concretos, a identificar las características específicas y 
esenciales de cada relato que lo diferencian de los otros. Por ello, es posible que en un mismo texto coexistan distintas visiones de la diversidad, aunque éste haya sido codificado en función de la visión considerada como prevalente.

No obstante, ambos relatos, como veremos, también comparten elementos discursivos respecto a la lógica pedagógica-funcionalista (Martín Criado, 2010) en la que se inscribe la mayoría de los textos sondeados. ${ }^{4}$ También debemos señalar la presencia de otras visiones más minoritarias o marginales en torno a la diversidad que se centran en las manifestaciones socioeconómicas de la desigualdad educativa y en la consideración de otras expresiones de la diversidad en el ámbito familiar y sexual, así como regional.

Finalmente, en cuanto al tipo de trabajos académicos que nutren a los diferentes relatos, si bien la mayoría de éstos son de carácter investigador (48,7\% en el caso del relato intercultural y $47,3 \%$ en el institucional), el relato institucional incorpora en mayor grado trabajos sobre experiencias pedagógicas (18,2\% frente a $6,8 \%$ del cultural).

Por su parte, el relato intercultural está compuesto por un conjunto de trabajos teóricos más numeroso (44,3\% frente a 34,5\% del institucional). Por otro lado, cabe señalar la preeminencia del uso de técnicas cuantitativas en los trabajos ubicados en el relato institucional (39\% frente al 23\% del intercultural) y de cualitativas en el cultural (61\% frente al 37\% de los artículos del relato institucional).

\section{El relato institucional: la diversidad como déficit y las necesidades educativas especificas como conformadoras de la diferencia}

El relato institucional, como apuntábamos, se apoya fundamentalmente en la definición de la diversidad establecida en la normativa educativa española, concretamente a partir de dos hitos legislativos: la Ley Orgánica General del Sistema Educativo (posteriormente, LOGSE) de 1990 y la LOE de 2006. De hecho, las justificaciones centrales para atender a la diversidad se sustentan principalmente en la aplicación de la legislación a la práctica educativa que "ha ido conformando un conjunto de recursos y respuestas pedagógicas que han tratado de atender a un alumnado cada vez más heterogéneo en capacidades, procedencia sociocultural y geográfica, motivación por el estudio y expectativas familiares de futuro" (Sales et al., 2010:2).

4 Las características de los espacios y actores que manejan los discursos sobre la diversidad así como sus contextos de producción han sido tratados en otro trabajo (Jiménez-Rodrigo y Guzmán-Ordaz, 2013). Indicamos aquí que la mayoría de estos textos se inscriben dentro de la tradición disciplinar de las Ciencias de la Educación. 
Aunque, a lo largo de su desarrollo, la LOE alude a diferentes situaciones - "diversidad de intereses, características y situaciones personales", "diversidad afectivo-sexual", "diversidad cultural y lingüística de las distintas Comunidades Autónomas", "dificultades de aprendizaje", "condiciones socioeconómicas desfavorables", "incorporación tardía al sistema educativo", "necesidades especiales"...-, "la atención a la diversidad" básicamente se refiere a aquellos alumnos y alumnas "que presentan necesidad específica de apoyo educativo".

En su Título II dedicado a la "Equidad en la Educación" se estipula cuáles son estas necesidades a partir de si el alumnado requiere "atención educativa diferente a la ordinaria, por presentar necesidades educativas especiales, por dificultades específicas de aprendizaje, por sus altas capacidades intelectuales, por haberse incorporado tarde al sistema educativo, o por condiciones personales o de historia escolar", de forma que "puedan alcanzar el máximo desarrollo posible de sus capacidades personales y, en todo caso, los objetivos establecidos con carácter general para todo el alumnado" (LOE, art. 71.2, 2006).

Los estudios inscritos dentro del relato institucional reproducen los criterios de distinción y clasificación del alumnado establecidos por la LOE. La mayoría de estos artículos (un 64\%) aborda las necesidades educativas específicas en relación con los déficits y problemas de aprendizaje del alumnado respecto a los criterios ordinarios.

Otro eje ampliamente considerado en los trabajos sobre diversidad educativa se refiere a las situaciones de discapacidad que pueden afectar al alumnado (un 23\%). Éste es categorizado como "alumnado que presenta necesidades educativas especiales” en referencia a: "sujetos que, por diversas causas -psíquicas, físicas, emocionales-, no pueden adaptarse, por completo, a una enseñanza normal.

A través del proceso educativo se procura que dichos sujetos puedan alcanzar la formación humana y la preparación necesaria para integrarse personal, social y profesionalmente en la sociedad a la que pertenecen" (Araque y Barrio, 2010: 5). Tanto en el caso de las necesidades específicas como en el de las especiales, la distinción de estos sujetos está delimitada y sujeta a la evaluación y diagnóstico profesional que determinarán los protocolos de atención relacionados con la adaptación y diversificación curricular o la educación especial (Araque y Barrio, 2010; Castaño, 2011; Toro, 2011).

Frente a las limitaciones en cuanto a capacidades del alumnado, el exceso de las mismas -situación denominada de diversas maneras: "sobredotación", "inteligencia exitosa", "altas capacidades"- también sustenta otro mecanismo de diferenciación educativa. Este aspecto, no obstante, es atendido por un número considerablemente reducido de estudios (un 5\%), muchos de ellos 
desde la Psicología. Pese a distinguir a estos niños y niñas por su superávit, paradójicamente, terminan siendo problematizados, no por su superior rendimiento, sino "por sus dificultades de aprendizaje y de integración escolar y social" materializadas en un alto porcentaje de "bajo rendimiento" y "fracaso escolar" entre el estudiantado "superdotado". Ese desajuste se explicaría por la falta de detección, diagnóstico y evaluación (Comes et al., 2009).

Por otro lado, y respecto a la "integración tardía en el sistema educativo español” —en alusión al alumnado de origen inmigrante extranjero ${ }^{5}$ — ésta es considerada en un exiguo porcentaje de artículos (un 3\%), siendo dicha situación problematizada a partir de una asignación anticipada de déficits curriculares acumulados como consecuencia de su "desconocimiento de la lengua vehicular del proceso de enseñanza-aprendizaje, el desfase escolar significativo y la integración del alumnado" (García García et al., 2012: 260).

A menudo, la construcción de la diferencia educativa del alumnado de origen extranjero va acompañada de imágenes deformadas que contribuyen a la reproducción de prejuicios etnicistas. ${ }^{6}$ Finalmente, se observa la presencia de una minoría de artículos (5\%) que, dentro de este relato institucional, aborda otros ejes de diferencia, fundamentalmente ligados a la diversidad familiar (en referencia a la pluralidad de formas familiares) y geográfica (en relación con las particularidades educativas regionales o de las zonas rurales).

Pese a la heterogeneidad de situaciones a las que estas diferentes categorías de alumnado se refieren, éstas se estructuran y funcionan básicamente en torno a la idea del déficit y la dicotomía normalidad $v s$. no normalidad. El uso de la diversidad se encuentra así fuertemente ligado a la ideología de la normalidad (Pérez de Lara, 2001), legitimadora de determinado orden, donde "no sólo se comienza a trabajar sobre la idea de clasificar lo normal distinguiéndolo de lo anormal, sino que se estructuran prácticas y saberes - disciplinas, instituciones, profesiones- encargados de normalizar al extenso grupo de sujetos que no cumplen las características deseables" (Almeida et al., 2010: 30).

5 Las dificultades de este tipo de alumnado que se señalan en la ley hacen referencia a "carencias lingüísticas o en sus competencias o conocimientos básicos”, que obstaculicen su escolarización, pero también respecto a sus familias en cuanto a la necesidad de "asesoramiento necesario sobre los derechos, deberes y oportunidades que comporta la incorporación al sistema educativo español" (LOE, art. 78, 2006).

6 Por ejemplo, que estos niños y niñas no han sido escolarizados en "su país de origen", que "viven en las calles" o "explotados en talleres"; u otros prejuicios acerca de su falta de motivación hacia lo escolar, los problemas de convivencia o la carencia de interés de sus familias. En este sentido, constatamos el papel de las élites académicas en la reproducción de discursos etnicistas y racistas (Van Dijk, 2009; Jiménez-Rodrigo et al., 2009). 
El alumnado definido como especial o con necesidades específicas a partir de la aplicación de determinados protocolos se convierte así en objeto de atención profesional especializada. Proceso que fácilmente desemboca en el etiquetaje $\mathrm{e}^{7}$ de quienes no siguen los niveles "ordinarios".

Frente a estas configuraciones prevalentes, se identifican dos derivaciones o deslizamientos discursivos del relato institucional en función de su focalización en las particularidades individuales o en las condiciones socioeconómicas que afectan al desigual rendimiento académico del alumnado.

Respecto al primer deslizamiento, observamos una tendencia significativa dentro del relato institucional a determinar la divergencia educativa a partir de las "diferencias individuales". Estos textos sostienen la pertinencia de concebir, en orden para alcanzar de forma efectiva la inclusión educativa, la "diversidad de todo el alumnado", "no sólo de los identificados con necesidad específica de apoyo educativo" (Vigo et al., 2010: 149).

De forma que "la atención a la diversidad" debería entender que todos los alumnos y alumnas son "especiales", señalando aquellas "diferencias que hacen que cada ser humano sea único" (Méndez y Del Pino, 2006). Se considera, en suma, que

cada alumno es diferente, por lo que la educación debe tratar las diferencias individuales (...). Por tanto, la atención a la diversidad consiste en aplicar un modelo de educación que reside en ser capaz de ofrecer a cada alumno la ayuda pedagógica que él necesite, ajustando la intervención educativa a la individualidad del alumnado: esta aspiración no es otra que adaptar la enseñanza a las diferentes capacidades, intereses y motivaciones del alumnado (Araque y Barrio, 2010: 11).

Tales particularidades, a menudo escasamente identificadas, hacen alusión frecuente a diferencias de motivación y "estilos de aprendizaje" (Arnaiz, 2008; Navarro, 2011). Esta manera de definir la diferencia educativa puede derivar en una psicologización, e incluso una biologización, del rendimiento académico en función de las capacidades o talentos de los individuos, lo que puede constituir una peligrosa derivación hacia propuestas individualistas meritocráticas ciegas a la consideración de la influencia del medio social.

Por otra parte, otro interesante deslizamiento se observa en ciertos textos donde la interpretación de las diferencias educativas conlleva una concepción más social. Ésta centra su atención en los factores sociales y económicos que generan no sólo diferencias sino también desigualdades entre grupos sociales y, en particular, entre el estudiantado en relación con sus trayectorias educa-

7 Es el caso de las etiquetas institucionalmente fijadas para identificar, diagnosticar y atender al alumnado con "NEE" o "ACNEES" ("alumnado con necesidades educativas especiales") y "ANEAE" o "ACNEAES" ("alumnado con necesidades específicas de apoyo educativo"). 
tivas y rendimientos. Son estudios - casi siempre cualitativos y teóricos- que focalizan su interés en la influencia de entornos sociales desfavorecidos y en los efectos excluyentes de las políticas de atención a la diversidad.

Resaltan, así, "la cara oculta de la diversidad en la escuela": el fracaso escolar padecido en los sistemas educativos por las personas "más diferentes y vulnerables" (Martínez, 2011: 167) y los problemas de segregación, desigualdad y exclusión social y educativa (Ballester y Vecina, 2011). También aluden a los efectos de etiquetaje y de estigmatización que generan los programas de diversificación educativa (Vega y Aramendi, 2009). El fracaso escolar, desde este marco, se define en términos más sociales que personales, por lo que reclaman la superación de interpretaciones individualistas para incorporar una mirada más amplia y comprehensiva de la problemática educativa.

Dentro de esta perspectiva institucional, las respuestas que se esperan y proponen a los agentes políticos e instituciones educativas se apoyan en la "atención" y "tratamiento" de quienes muestran algún tipo de dificultad o necesidades educativas. Según los textos analizados, estas medidas están orientadas a una mejora de la calidad y eficacia de la educación que habría de traducirse en una elevación del rendimiento académico y de la promoción estudiantil de quienes presentan algún tipo de hándicap escolar.

Por ello, adquieren especial protagonismo las innovaciones e intervenciones pedagógicas a nivel de aula y, especialmente, las ligadas a asignaturas de ciencias naturales y experimentales que potencien las capacidades y las habilidades del estudiantado. Junto a la búsqueda de la mejora del rendimiento escolar, los textos también recogen constantes alusiones a la inclusión educativa como fórmula para potenciar la eficiencia educativa en la respuesta de los centros frente a la diversidad de su alumnado (Barrio, 2009; García García et al., 2012).

Se entiende así que la atención a la diversidad puede facilitar el desarrollo de una "educación única, igualitaria y de calidad [...] que rechace cualquier tipo de exclusión educativa y que potencie la participación y el aprendizaje equitativo" (Araque y Barrio, 2010: 4).

\section{El relato intercultural: la inmigración extranjera y las diferencias culturales como fuente de diversidad}

El relato intercultural de la diversidad identificado en el examen de la literatura española se apoya en la necesidad de responder desde la escuela al hecho inmigrante y, en concreto, a la llegada "intensa", "en progresivo aumento", "masiva” a las aulas de alumnado de origen extranjero (García Medina, 2006; 
Herrada, 2009; García Fernández et al., 2010), reproduciendo también en estas justificaciones tópicos comunes que han rodeado la construcción de la inmigración no comunitaria como problema en España (Santamaría, 2002; Gijón et al., 2006).

De tal forma, la inmigración -o más precisamente, determinada inmigración y determinados grupos inmigrantes- se convierte en el principal leitmotiv para proponer el cambio educativo:

Nuestra tierra ha pasado en las últimas décadas de ser un país de emigrantes a convertirse en país de acogida de inmigrantes de diversos lugares, etnias, costumbres, lenguas y religiones. Por su parte, y centrándonos en el contexto educativo, es evidente la existencia de aulas multiculturales y del fenómeno multicultural entendido como la pluralidad de alumnos de procedencias diversas en espacios educativos comunes (Leiva, 2011: 5).

Sin embargo, no deja de ser paradójico que este descubrimiento del fenómeno de la multiculturalidad se haya producido a espaldas del reconocimiento de otras diferencias culturales que han venido caracterizando las aulas españolas. De hecho, sólo un $6 \%$ de los textos ubicados dentro de este relato aborda como sujeto cultural diferencial a la población gitana, minoría étnica con mayor y más antigua presencia en España y con graves problemas de marginación y exclusión.

Prácticamente la totalidad de trabajos se focaliza en la inmigración extranjera, aunque de forma limitada y parcial al centrarse en determinadas nacionalidades o procedencias relativas a "países en desarrollo" o "extracomunitarios". La inmigración y la multiculturalidad asociada exigen, entonces, de la comunidad educativa nuevas estrategias de adaptación e inclusión de estos nuevos sujetos culturalmente dispares (García Velasco, 2009).

Dentro de este relato de la diversidad, la diferencia cultural se articula primordialmente sobre la pertenencia, o no, a determinados orígenes geográficos o nacionales. La cultura se reduce fundamentalmente a la "marca" de la nacionalidad - propia o familiar- materializándose en determinadas variables más patentes y tangibles, como la lengua, la religión o determinados rasgos físicos.

Las categorías "inmigrante" y/o "extranjero" se establecen como indicadores clave de configuración de las diferencias culturales, entendiéndose en muchas ocasiones de forma determinista, estanca, estática y ligadas inevitablemente a su procedencia, como un peso del que no pueden despojarse los sujetos incluso habiendo adquirido, por ejemplo, la nacionalidad española o simplemente habiendo nacido en España (la denominación "segunda generación" hace alusión a esta marca indeleble). 
Esta visión de la diversidad y de la alteridad cultural contrasta con la de los relatos sobre la diversidad elaborados en otros contextos, como el latinoamericano, donde los ejes centrales se apoyan más en la condición rural e indígena (Ibáñez et al., 2012) o el estadounidense focalizado en la diferencia racial (Ahmed, 2007; Berrey, 2011; Marvasti y McKinney, 2011; Bhopal y Rhamie, 2014).

La definición de la diversidad desde este relato intercultural arroja, en suma, una visión esencializada y homogeneizadora de la cultura, que afecta tanto a la considerada "autóctona" como a la atribuida a los otros sujetos o grupos procedentes de otros espacios nacionales. Se reproduce el "mito de la consistencia interna de las culturas", donde las culturas representan comunidades homogéneas de creencias y estilos de vida, de forma que "la diversidad cultural se transforma en una categoría ontológica: supone el reconocimiento de contenidos y costumbres culturales preestablecidos exentos de mezcla y contaminación" (Duschatzky y Skliar, 2001: 197).

Esta separación entre culturas se establece fundamentalmente en términos negativos y excluyentes: "no nacional", "no autóctono", en definitiva, "no de aquí", "no como nosotros", ignorando en muchos casos las diferencias culturales y desigualdades dentro del colectivo inmigrante extranjero, ya sea por procedencia, edad, género o clase social. De hecho, es común la indeterminación en los textos sobre los orígenes geográficos o nacionales del alumnado al que se pretende atender en su diversidad.

Son pocos los estudios donde se apuntan o especifican las procedencias concretas, aunque en gran parte de éstos responden a los criterios estadísticos de clasificación que manejan las agencias administrativas o a amplias clasificaciones geográficas, por ejemplo, en función del continente o "área cultural". No obstante, hay un tipo de discentes culturalmente diverso que atrae buena parte de la atención de los trabajos que sí parecen contemplar las procedencias concretas del alumnado y es el "alumnado latinoamericano", categoría que opera como gran contenedor y aglutinador de diferencias culturales, y que no está exenta de interpretaciones estereotípicas y simplificadoras de estas "otras culturas", "identidades culturales" y sus sistemas educativos.

De este modo, en muchos de los textos analizados se observa, como afirma Gunther Dietz (2008: 20), una tendencia a "problematizar" implícitamente la existencia de diversidad cultural en el aula, "importando" sin sentido crítico conceptos básicos de la antropología, tales como "cultura", "grupo étnico" y "etnicidad" en sus definiciones a menudo decimonónicas y ya caducas", lo que lleva también a la etnificación de las diferencias culturales mediante la cosificación de sus portadores. 
Estos procesos de categorización del distinto, como ha señalado Eduardo Menéndez (2002: 109), implican "ignorar al sujeto o pensarlo en términos de identidad casi indistinguible del grupo local, de la etnia, de la comunidad de pertenencia, de tal manera que el sujeto adquiría/expresaba los rasgos de estas unidades consideradas como homogéneas, integradas coherentes, auténticas, etc., que caracterizan simultáneamente a la cultura y a su sujeto".

La articulación de la diversidad a partir de la dicotomía "nosotros"/ "otros" tiene efectos homogeneizantes y globalizadores al enmascarar la heterogeneidad del colectivo "inmigrante", no sólo en cuanto a nacionalidad u origen geográfico sino también en cuanto a diferencias culturales entre regiones o grupos, por ejemplo, y otros factores estructurales y condiciones que operan interseccionalmente ${ }^{8}$ con la nacionalidad/raza/etnia como el género, clase social, edad, orientación sexual o capacidades psicofísicas.

Pocos textos de los explorados -y elaborados desde una perspectiva cualitativa y teórica-reflexiva- plantean una visión crítica en torno a la simple explicación de la diferencia escolar en función de "meros atributos inherentes comunitarios", reivindicando al mismo tiempo el papel del contexto sociohistórico y de la desigualdad estructural (Franzé, 2008).

Critican los efectos asimilacionistas, discriminatorios y segregadores de una parte del alumnado que se extraen de una interpretación exclusivamente culturalista de la diferencia: "la diversidad no es sólo una realidad social abordable desde la situación de posiciones e identidades marginalizadas; sino que ha de ser abordada desde una teoría general que dé cuenta de la forma en que acontecen los procesos de subjetivación, como ejercicios articulatorios transidos por desiguales y jerarquizadas relaciones de poder" (Madero et al., 2011: 146).

Advierten también de "la contradicción que supone hablar de una diversidad en la que cabemos todos y, a su vez, proponer una atención a la diversidad", donde los diversos son los otros, los diferentes, los desplazados de la "normalidad". "[...] los extranjeros, los extraños, los que no siguen el patrón común de la mayoría, el que 'debe ser”" (Sánchez, 2011: 145).

8 El análisis interseccional busca salvar las trampas esencialistas de definiciones unidireccionales y argumentos dicotómicos, considerando la construcción de las experiencias sociales mediante la intersección de múltiples diferencias, sean de género, étnica, racial de clase, nacional, conllevando la ruptura de un ideal hegemónico de un "nosotros" homogéneo (Guzmán-Ordaz, 2015). Igualmente, el examen de otros tipos de aportaciones teóricas y metodológicas más distantes de los estándares positivistas académicos podría contribuir a ampliar la mirada hacia otros entendimientos más críticos con los planteamientos esencialistas prevalentes. 
Frente a la visión institucional que valora la diversidad en términos de carencia y de problema para la labor educativa, la visión cultural va acompañada de una percepción marcadamente optimista de la diversidad como un “reto", un "valor positivo" y una "riqueza" (Martín Rojo, 2007; Leiva, 2011). La incorporación de las diferencias culturales al proceso educativo se entiende aquí como una oportunidad de enriquecimiento (Herrada, 2009).

Estas dispares valoraciones se observan, a modo de botón de muestra, en el caso de la lengua del alumnado inmigrante extranjero. Frente al enfoque institucional que evalúa la diferencia lingüística en términos de déficit curricular en tanto falta de conocimiento de la lengua "autóctona”, el enfoque intercultural aprecia el conocimiento de otras lenguas en términos de competencias culturales: "sobre todo, los recursos lingüísticos que caracterizan a estos niños y jóvenes constituyen un valioso capital tanto individual como colectivo en este mundo crecientemente globalizado" (García-Cano et al., 2010: 292).

Las distancias entre estos dos relatos a la hora de entender la diversidad también se observan en las respuestas que los artículos ubicados dentro de este último enfoque esbozan frente a los "desafíos" planteados por el "alumnado inmigrante” y la multiculturalidad extranjera. Éstas se basan en las herramientas de la educación intercultural, donde la convivencia pacífica, el reconocimiento y el respeto de las diferencias culturales se conforman como metas centrales.

Con estos propósitos, son comunes los proyectos y planes transversales de centro que pretenden mejorar las relaciones y la interacción entre el alumnado "culturalmente diferente", adquiriendo gran importancia las asignaturas humanísticas transmisoras de valores. La interculturalidad se entendería como "una verdadera y efectiva interacción entre culturas que nos haga conocernos y re-conocernos a través de este conocimiento previo basado en el respeto".

El otro no es un elemento contaminador sino enriquecedor que, sin duda, hay que fomentar desde todos los ámbitos sociales, entre ellos el educativo, con una "atención a la diversidad" que tenga en cuenta todas y cada una de las pertenencias presentes tanto en el aula como fuera de ella (Sánchez, 2011: 151). Sobre todo, la clave del cambio se encuentra no en fomentar las competencias individuales como en el enfoque institucional, sino en "favorecer las interacciones entre individuos con diferentes sistemas sociales y culturales que entran en contacto en un mismo contexto" (Ayora, 2010: 45).

La interculturalidad se presupone así como el canal idóneo para hacer que las diferencias culturales fluyan dentro del sistema educativo que, por otro lado, lleva la responsabilidad intrínseca de generar un clima de convivencia y aprendizaje igualitario y, por ende, inclusivo, solidario, e incluso en algunas 
lecturas, calificado como "emancipador" (Hernández de la Torre, 2009; Leiva, 2011). Observamos cómo frente a un problema culturizado la respuesta inevitablemente también se culturiza, lo cual conlleva una reinterpretación de las desigualdades socioeconómicas, legales y/o políticas como diferencias culturales (Dietz, 2012).

No obstante, y en convergencia con el enfoque institucional, es en la adecuada formación inicial y permanente de su profesorado donde recaen las principales soluciones frente a los "retos" de la diversidad. La escuela se piensa como un sistema autónomo, autosuficiente y autorreferencial y se presenta al profesorado como principal protagonista, "elemento esencial" de los cambios educativos y sociales, al dirigir los procesos de socialización que garantizarían la cohesión social y cultural.

Se reclama en ambos relatos una respuesta sustentada en la transformación curricular y en la mejora de la formación docente: más en términos de competencias, motivación y recursos educativos en el institucional, más en términos de actitudes y sensibilidad en el cultural. Sendos enfoques sintonizarían en torno a los elementos centrales del "discurso pedagógico habitual" -la preminencia didáctica, la insistencia de los recursos y la focalización en los procesos intraescolares- (Villar y Hernández, 2013) y de las premisas idealistas que sustentan a la mayor parte de las reformas escolares (Martín Criado, 2004).

\section{Conclusiones}

El análisis de la producción científica española en torno a la diversidad en el ámbito escolar ha permitido caracterizar, a partir de la identificación de sus configuraciones narrativas, dos relatos hegemónicos. Por una parte, encontramos un relato institucional sustentado en la definición normativa de la diversidad educativa en función de las diferentes capacidades y rendimientos escolares. Y, por otra, un relato intercultural elaborado a partir de la consideración de la inmigración extranjera como principal factor de diversidad cultural en las aulas.

Aunque ambos relatos seleccionan distintas características de clasificación y jerarquización, se observan mecanismos comunes de construcción de la diferencia. Primero, éstos se dirigen primordialmente hacia el alumnado que se convierte en depositario de determinadas características personales o culturales que lo definen como diferente, como diverso. Segundo, estas características se definen y se atribuyen a través de un procedimiento de reproducción dicotómica de fronteras de inclusión y exclusión en relación con lo señalado 
como normalidad, ya sea en términos de rendimientos educativos, ya sea en términos de adscripción geográfica-cultural.

De esta forma, y paradójicamente, la diversidad termina planteándose en términos de "oposición a totalidades de normalidad", contribuyendo así a "la garantía de identidades fijas, centradas, homogéneas, estables” (Duschatzky y Skliar, 2001: 189).

La construcción de la diferencia desde determinados parámetros educativos y culturales de forma dicotómica y excluyente también tiene efectos enmascaradores de situaciones de desigualdad, segregación y desventaja a partir del énfasis de ciertos rasgos que definen y hacen diversa a una población (Duschatzky y Skliar, 2001; Ramos, 2012). De tal forma, en estos dos enfoques preponderantes también se observa en una mayoría de trabajos la omisión e infravaloración del contexto social y económico en la determinación de las diferencias y desigualdades en el ámbito escolar.

En el institucional, sobre todo, se enfatizan las diferencias individuales en relación con la heterogeneidad de capacidades, rendimientos y trayectorias educativas, llegando incluso a observarse ciertas interpretaciones biologicistas de la diferencia educativa. En el cultural, en cambio, las particularidades del sujeto quedan desdibujadas a partir de una atribución esencialista y homogeneizadora de determinadas características del grupo cultural al que se adscribe con base en su condición inmigrante extranjera.

No obstante, cabe señalar la emergencia en ambas configuraciones de deslizamientos marginales hacia posiciones críticas que cuestionan el carácter esencialista del discurso preponderante sobre la diversidad en la escuela. Son estudios -muchos de ellos elaborados desde metodologías cualitativas y perspectivas teóricas sensibles a la desigualdad-que resaltan la importancia de lo socioeconómico y la necesidad de contemplar las interacciones de las diferencias educativas y culturales con otros factores de diferencia y desigualdad, como la clase social, el género, la etnicidad o el territorio.

Estos resultados no pueden entenderse sin atender al contexto de producción de los textos -fundamentalmente dentro de la tradición disciplinar pedagógica- y al tipo de producto académico analizado - artículos indexados según los estándares temáticos y metodológicos que rigen el campo académico-. Por ello, una línea futura de investigación podría enfocarse hacia el estudio de la emergencia de nuevas posiciones discursivas críticas respecto a la diversidad.

En todo caso, y pese a las intenciones declaradas en algunos trabajos, aún no se ha consolidado la superación de la referencia a categorías binarias, así como la consideración de otros instrumentos conceptuales para pensar la 
diversidad en un contexto que apueste más por las intersecciones (GuzmánOrdaz y Márquez-Lepe, 2012). Y ésta es una cuestión importante, porque dependiendo de cómo se definan y legitimen académica y socialmente las etiquetas que marcan a "los diferentes" así como las distancias que nos separan de éstos, se definirán y legitimarán los mecanismos de relación y de transacción social entre los grupos (Menéndez, 2002).

Esto se materializa consecuentemente en la vinculación entre los discursos sobre la diversidad y las políticas de reconocimiento de la diferencia de las minorías, y que proyectan una idea de una sociedad que necesariamente debe incluir, aceptar y respetar las diferencias de todos los que la constituyen (Almeida et al., 2010).

El peligro del relativismo planea así como una sombra en los discursos sobre la diversidad que asumen la admisión de lo diferente como principio (Duschatzky y Skliar, 2001). También se advierte de las paradojas que conllevan las políticas de la diferencia en su reivindicación de los particularismos en un marco socioeconómico cada vez más fragmentado y excluyente (Alonso, 2005).

En suma, la reflexión sobre los usos de la diversidad y los debates que éstos concitan no pueden limitarse sólo a los procedimientos de construcción, reconocimiento o celebración de la diferencia, sino también, y sobre todo, a sus implicaciones sociales en cuanto a esas "grietas" y "contornos" -como señaló Geertz- que marcan nuestra relación con los otros y las dinámicas de integración social en un mundo desigual.

\section{Bibliografía}

Ahmed, Sara (2007), “The language of diversity", en Ethnic and Racial Studies, vol. 30, núm. 2, UK: University of Surrey.

Almeida, María Eugenia et al. (2010), "Nuevas retóricas para viejas prácticas. Repensando la idea de diversidad y su uso en la comprensión y abordaje de la discapacidad", en Politica y Sociedad, vol. 47, núm. 1, España: Universidad Complutense de Madrid.

Alonso, Luis Enrique (2005), “¿Redistribución o reconocimiento? Un debate sociológicamente no siempre bien planteado", en Ariño, Antonio [ed.], Las encrucijadas de la diversidad cultural, España: CIS.

Alonso, Luis Enrique y Carlos J. Fernández (2006), "Roland Barthes y el análisis del discurso", en Empiria. Revista de Metodología de las Ciencias Sociales, núm. 12, España: UNED.

Araque, Natividad y José Luis Barrio (2010), "Atención a la diversidad y desarrollo de procesos educativos inclusivos", en Prisma Social. Revista de Ciencias Sociales, núm. 4, España: Fundación iS+ D para la Investigación Social Avanzada.

Arnaiz, Pilar (2008), "Indicadores de calidad para la atención a la diversidad del alumnado en la educación secundaria obligatoria”, en Revista de Educación, núm. 349, España: Ministerio de Educación Cultura y Deporte. 
Convergencia Revista de Ciencias Sociales, núm. 71, 2016, Universidad Autónoma del Estado de México

Ayora, María del Carmen (2010), "Diversidad lingüística y cultural en un ámbito educativo de lenguas de contacto”, en Pragmalingüistica, núm. 18, España: Universidad de Cádiz.

Ballester, Lluís y Carlos Vecina (2011), "Intervención comunitaria, diversidad y complejidad social. El problema de la segregación social en la escuela”, en Prisma Social. Revista de Ciencias Sociales, núm. 6, España: Fundación iS+ D para la Investigación Social Avanzada.

Barrio, José Luis (2009), “Hacia una educación inclusiva para todos”, en Revista Complutense de Educación, vol. 20, núm. 1, España: Universidad Complutense de Madrid.

Berger, Peter y Thomas Luckmann (2006), La construcción social de la realidad, España: Amorrortu.

Berrey, Ellen C. (2011), "Why diversity became orthodox in higher education, and how it changed the meaning of race on campus", en Critical Sociology, vol. 37, núm. 5, UK: Association for Critical Sociology.

Bhopal, Kalwant y Rhamie, Jasmine (2014), "Initial teacher training: understanding 'race', diversity and inclusion”, en Race Ethnicity and Education, vol. 17, núm. 3, UK: University of Birminghan.

Carrasco, Concepción (2015), "Discurso de futuros docentes acerca de la diversidad intercultural”, en Papers, vol. 100, núm. 2, España: Universidad Autónoma de Barcelona.

Castaño, Raimundo (2011), "El currículum y a la atención a la diversidad en las etapas de Educación Básica, Primaria y Secundaria Obligatoria, en el marco de la Ley Orgánica de Educación", en Hekademos. Revista Educativa Digital, núm. 6, España: Asociación AFOE.

Colectivo IOE (1997), La diversidad cultural y la escuela. Discurso sobre atención a la diversidad con referencia especial a las minorias étnicas de origen extranjero, España: CIDE.

Coll, César (2002), "La atención a la diversidad en el proyecto de Ley de Calidad de la Educación o la consagración del orden natural de las cosas", en Aula de Innovación Educativa, núm. 115, España: Grao Publicaciones.

Comes, Gabriel et al. (2009), "Análisis de la legislación española sobre la educación del alumnado con altas capacidades”, en Escuela Abierta, núm. 12, España: Fundación San Pablo Andalucía.

Conde, Fernando (2009), Análisis sociológico del sistema de discursos, España: Colección Cuadernos metodológicos, núm. 43, Centro de Investigaciones Sociológicas.

Coronel José M. e Inmaculada Gómez-Hurtado (2015), “Nothing to do with me! Teachers' perceptions on cultural diversity in Spanish secondary schools", en Teachers and Teaching, vol. 21, núm. 4, UK: International Study Association on Teachers and Teaching.

Dietz, Gunther (2008), "El paradigma de la diversidad cultural: tesis para el debate educativo", en IX Congreso Nacional de Investigación Educativa. Conferencias Magistrales: México.

Dietz, Gunther (2012), Multiculturalismo, interculturalidad y educación: una aproximación antropológica, México: Fondo de Cultura Económica.

Duschatzky, Silvia y Carlos Skliar (2001), "En nombre de los otros. Narrando a los otros en la cultura y en la educación”, en Larrosa, Jorge y Carlos Skliar [eds.], Habitantes de Babel: Políticas y poéticas de la diferencia, España: Laertes. 
María Luisa Jiménez-Rodrigo y Raquel Guzmán-Ordaz. Definiendo a los otros: relatos académicos sobre la diversidad en la escuela

Franzé, Adela (2008), "Diversidad cultural en la escuela. Algunas contribuciones antropológicas", en Revista de Educación, núm. 345, España: Ministerio de Educación, Cultura y Deporte.

García Cano, María et al. (2010), "Estrategias bilingües e interculturales en familias transmigrantes”, en Revista de Educación, núm. 352, España: Ministerio de Educación, Cultura y Deporte.

García Fernández, José Antonio et al. (2010), “Estudio del sistema y funcionamiento de las aulas de enlace de la Comunidad de Madrid. De la normativa institucional a la realidad cotidiana”, en Revista de Educación, núm. 352, España: Ministerio de Educación, Cultura y Deporte.

García García, Mercedes et al. (2012), "Medidas eficaces en atención a la diversidad cultural desde una perspectiva inclusiva”, en Revista de Educación, núm. 358, España: Ministerio de Educación, Cultura y Deporte.

García Medina, Raúl (2006), “Un enfoque educativo piagetiano desde la práctica docente: en tomo a la adquisición del concepto de número por alumnos con discapacidad cognitiva”, en Tendencias Pedagógicas, núm. 11, España: Universidad Autónoma de Madrid.

García Velasco, Alba (2009), "La integración del alumnado inmigrante en el ámbito escolar: adecuar los recursos y aprovechar la diversidad”, en Cuadernos de Trabajo Social, vol. 22, España: Universidad Complutense de Madrid.

Geertz, Clifford (1996), Los usos de la diversidad, España: Paidós.

Gimeno, José (2000), "La construcción del discurso acerca de la diversidad y sus prácticas", en Aula de Innovación Educativa, núm. 81-82, España: Grao Publicaciones.

Gijón Sánchez, M. Teresa et al. (2006), "Más allá de la diferencia, tras el cristal de la diversidad. La inmigración en la literatura biomédica en España”, en Fernández Juárez, Gerardo [coord.], Salud e interculturalidad en América Latina. Antropología de la salud y critica intercultural, Ecuador-España: Abba-Yala.

Guzmán-Ordaz, Raquel (2015), "El paradigma interseccional: rutas teóricas-metodológicas para el análisis de las desigualdades sociales", en Saletti-Cuesta, Lorena [coord.], Traslaciones en los estudios feministas, Málaga: Perséfone-Universidad de Málaga.

Guzmán-Ordaz, Raquel y Esther Márquez-Lepe (2012), "Intersectionality as a Research Strategy for Diversity in Education 'field' and Migration Process”, en IAIE International Conference: Tapelewilis for Intercultural Education: Sharing Experiences, building alternatives: Veracruz, México.

Hernández de la Torre, Elena (2009), "Una educación entre culturas en el punto de mira de la atención a la diversidad", en Revista de Educación Inclusiva, vol. 2, núm. 2, España: Universidad de Jaén.

Herrada, Rosario (2009), "Mosaicos conceptuales vinculados a la diversidad cultural de estudiantes universitarios de magisterio: más que palabras”, en Docencia e Investigación, año 34, núm. 19, España: Universidad de Castilla La Mancha.

Ibáñez Salgado, Nolfa et al. (2012), "La comprensión de la diversidad en interculturalidad y educación”, en Convergencia: Revista de Ciencias Sociales, núm. 59, México: Universidad Autónoma del Estado de México.

Jiménez-Rodrigo, María Luisa et al. (2009), "El análisis de la literatura biomédica en España en clave de diversidad cultural y de género", en Empiria, núm. 17, España: UNED. 
Jiménez-Rodrigo, María Luisa y Raquel Guzmán-Ordaz (2013), "Sociología de la construcción de los conceptos académicos: el caso de la 'diversidad' en educación”, en Sociología Histórica, núm. 2, España: Universidad de Murcia.

Lawson, Hazel, Boyask, Ruth y Sue Waite (2013), "Construction of difference and diversity within policy and practice in England", en Cambridge Journal of Education, vol. 43, núm. 1, UK: University of Cambridge.

Leiva, Juan José (2011), "Fundamentos Pedagógicos de la Educación Intercultural: construyendo una cultura de la diversidad para una escuela humana e inclusiva”, en Miscelánea Comillas, vol. 69, núm. 134, España: Universidad Pontificia de Comillas.

LOE. Ley Orgánica 2/2006, de 3 de mayo, de Educación (2006), España: Gobierno de España.

Madero, Beatriz et al. (2011), "Repensando la diversidad en la escuela”, en Revista de Estudios de Juventud, vol. 11, núm. 95, España: INJUVE.

Martín Criado, Enrique (2004), "El idealismo como programa y como método de las reformas escolares", en El nudo en la red, núm. 3-4, España: Coordinadora de Asociaciones Culturales de Madrid.

Martín Criado, Enrique (2010), La escuela sin funciones. Crítica de la sociología de la educación critica, España: Bellaterra.

Martín Rojo, Luisa (2007), “'Sólo en español': una reflexión sobre la norma monolingüe y la realidad multilingüe en los centros escolares”, en Revista de Educación, núm. 343, España: Ministerio de Educación, Cultura y Deporte.

Martínez Domínguez, Begoña (2011), "Luces y sombras de las medidas de atención a la diversidad en el camino de la inclusión educativa", en Revista Interuniversitaria de Formación del Profesorado, vol. 25, núm. 1, España: AUFOP.

Marvasti, Amyr y Mckinney, Karyn (2011), “Does diversity mean assimilation?”, en Critical Sociology, vol. 37, núm. 5, UK: Association for Critical Sociology.

Méndez, María Félix e Inmaculada del Pino (2006), "La atención a la diversidad en educación física. Propuestas de actuación docente”, en Efsdeportes.com, Revista Digital, año 11, núm. 97, España: Universidad de Barcelona.

Menéndez, Eduardo (2002), La parte negada de la cultura. Relativismo, diferencias y racismo, España: Bellaterra.

Monsalvo, Eugenio y Miguel Ángel Carbonero (2005), "La atención a la diversidad como ideología educativa”, en Revista Psicodidáctica, vol. 10, núm. 1, España: Universidad del País Vasco.

Nader, Laura (1972), “Up the anthropologist. Perspectives gained from studying up”, en Dell Hymes [coord.], Reinventing Anthropology, New York: Vintage Books.

Navarro, Manuel (2011), "Medidas educativas y organizativas de atención a la diversidad que mejoran el rendimiento escolar en el IES Sierra de los Filabres de Serón (Almería)", en Espiral. Cuadernos del profesorado, vol. 4, núm. 7, España: Centro de Profesorado de Cuevas Olula.

Pérez de Lara, Nuria (2001), "Identidad, diferencia y diversidad: mantener viva la pregunta", en Jorge Larrosa y Carlos Skliar [eds.], Habitantes de Babel: Politicas y poéticas de la diferencia, España: Laertes.

Ramos, José Antonio (2012), "Cuando se habla de diversidad ¿de qué se habla? Respuestas desde el sistema educativo", en Revista Interamericana de Educación de Adultos, año 34, núm. 1, México: OEA. 
María Luisa Jiménez-Rodrigo y Raquel Guzmán-Ordaz. Definiendo a los otros: relatos académicos sobre la diversidad en la escuela

Sales, Auxiliadora, Odet Moliner y Lidón Moliner (2010), "Estudios de la eficacia académica de las medidas específicas de Atención a la Diversidad desde la percepción de los implicados”, en Estudios sobre Educación, núm. 19, España: Universidad de Navarra.

Sánchez Rojo, Alberto (2011), "Raimón Panikkar va a la escuela: diálogo intercultural y atención a la diversidad”, en Bajo Palabra. Revista de Filosofía, II época, núm. 6, España: Universidad Autónoma de Madrid.

Santamaría, Enrique (2002), La incógnita del extraño. Una aproximación a la significación sociológica de la "inmigración no comunitaria”, España: Anthropos.

Skliar, Carlos (2005), "Poner en tela de juicio la normalidad, no la anormalidad. Políticas y falta de políticas en relación con las diferencias en educación”, en Revista Educación y Pedagogía, vol. 17, núm. 41, Colombia: Universidad de Antioquia.

Terrén, Eduardo (2001), "La conciencia de la diferencia étnica: identidad y distancia cultural en el discurso del profesorado”, en Papers, núm. 63/64, España: Universidad de Barcelona.

Toro, Laia (2011), "Uso de perfiles de personalidad para la atención a la diversidad: evidencias en alumnos de educación especial", en Educación y diversidad. Revista Interamericana de Investigación sobre Discapacidad e Interculturalidad, vol. 4, núm. 1, España: Universidad de Zaragoza.

Van Deventer Iverson, Susan (2007), "Camouflaging power and privilege: A critical race analysis of university diversity policies", en Educational Administration Quarterly, vol. 43, núm. 5, USA: University Council for Educational Administration.

Van Dijk, Teun A. (2009), Discurso y Poder, España: Gedisa.

Vega, Amando y Pello Aramendi (2009), "La atención a la diversidad: interrogantes para la iniciación profesional de los 'fracasados", en Enseñanza and Teaching, núm. 27, España: Universidad de Salamanca.

Vigo, Begoña et al. (2010), "Preparando profesores para la atención a la diversidad: potencialidades y limitaciones de un proyecto de innovación y mejora interdisciplinar", en Revista Interuniversitaria de Formación de Profesorado, vol. 24, núm. 3, España: Universidad de Zaragoza.

Villar, Alicia y Francesc Jesús Hernández (2013), “Anomalías sociológicas en el discurso pedagógico”, en Praxis Sociológica, núm. 17, España: Universidad de Castilla La Mancha. Zapata, Ricard (2008-2009), "Diversidad y política pública”, en Nuria del Viso [coord.], Dossier Reflexiones sobre las diversida(des), España: Centro de Investigación para la Paz (CIP-Ecosocial). 


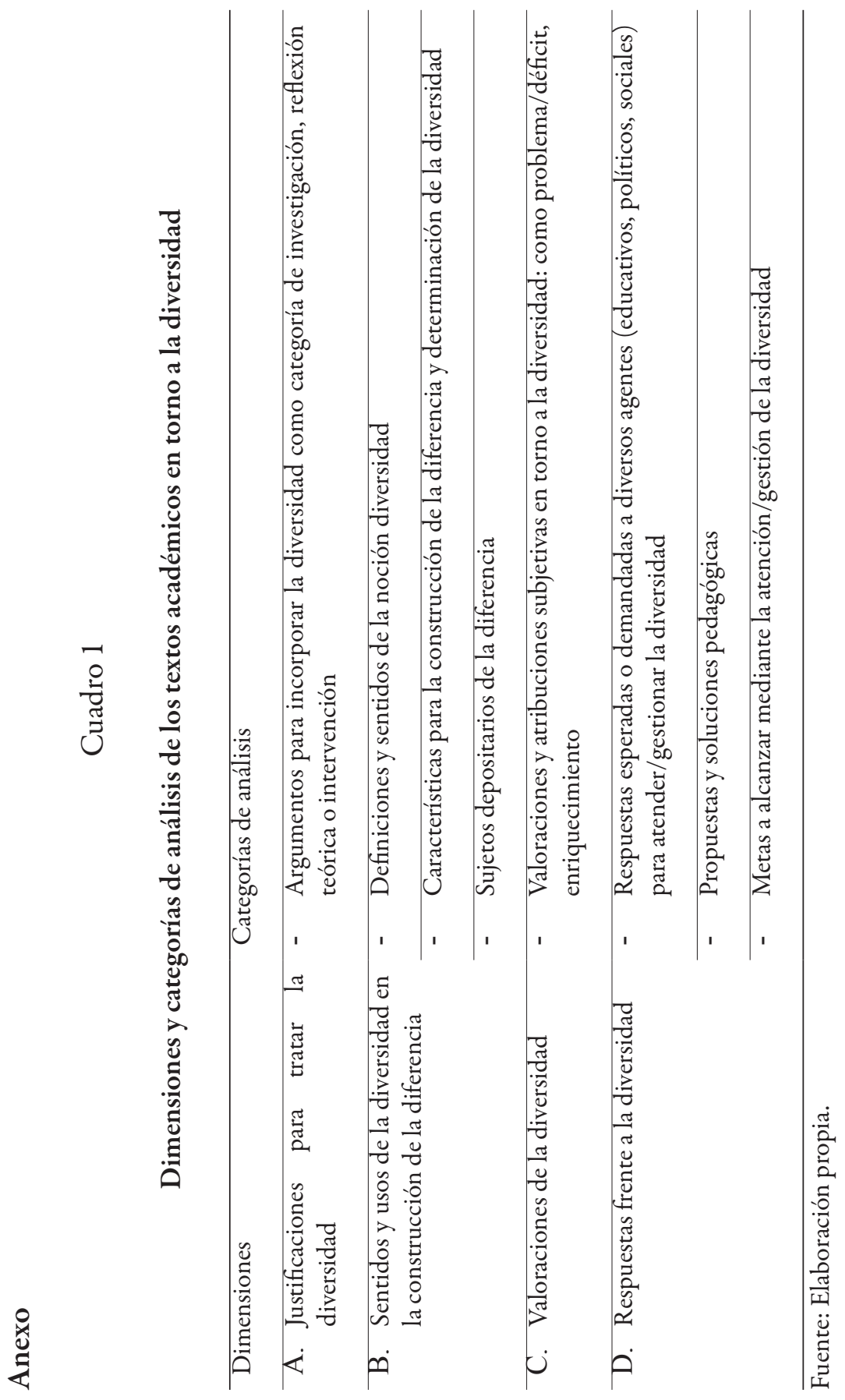




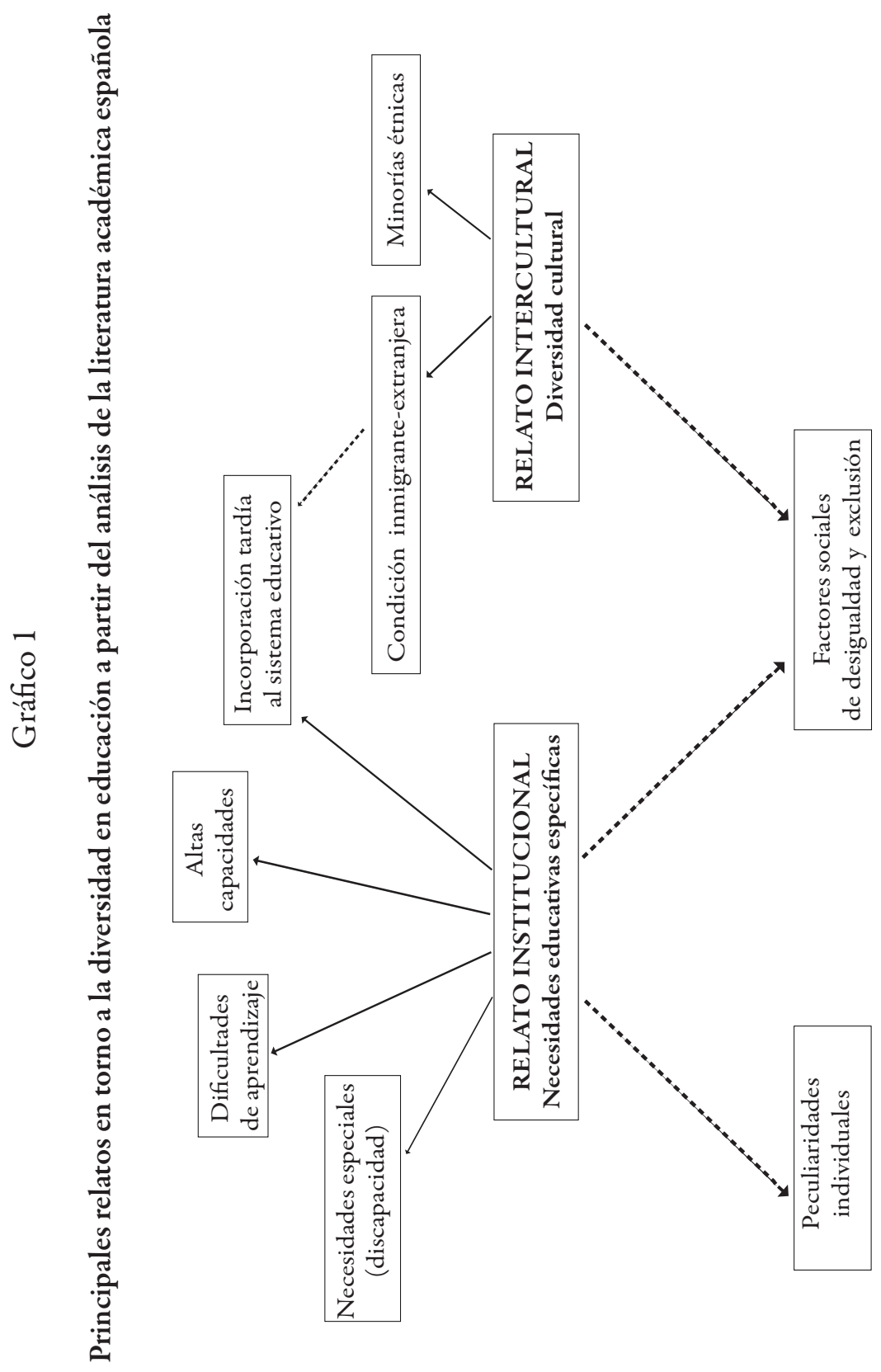

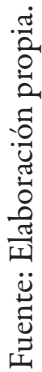




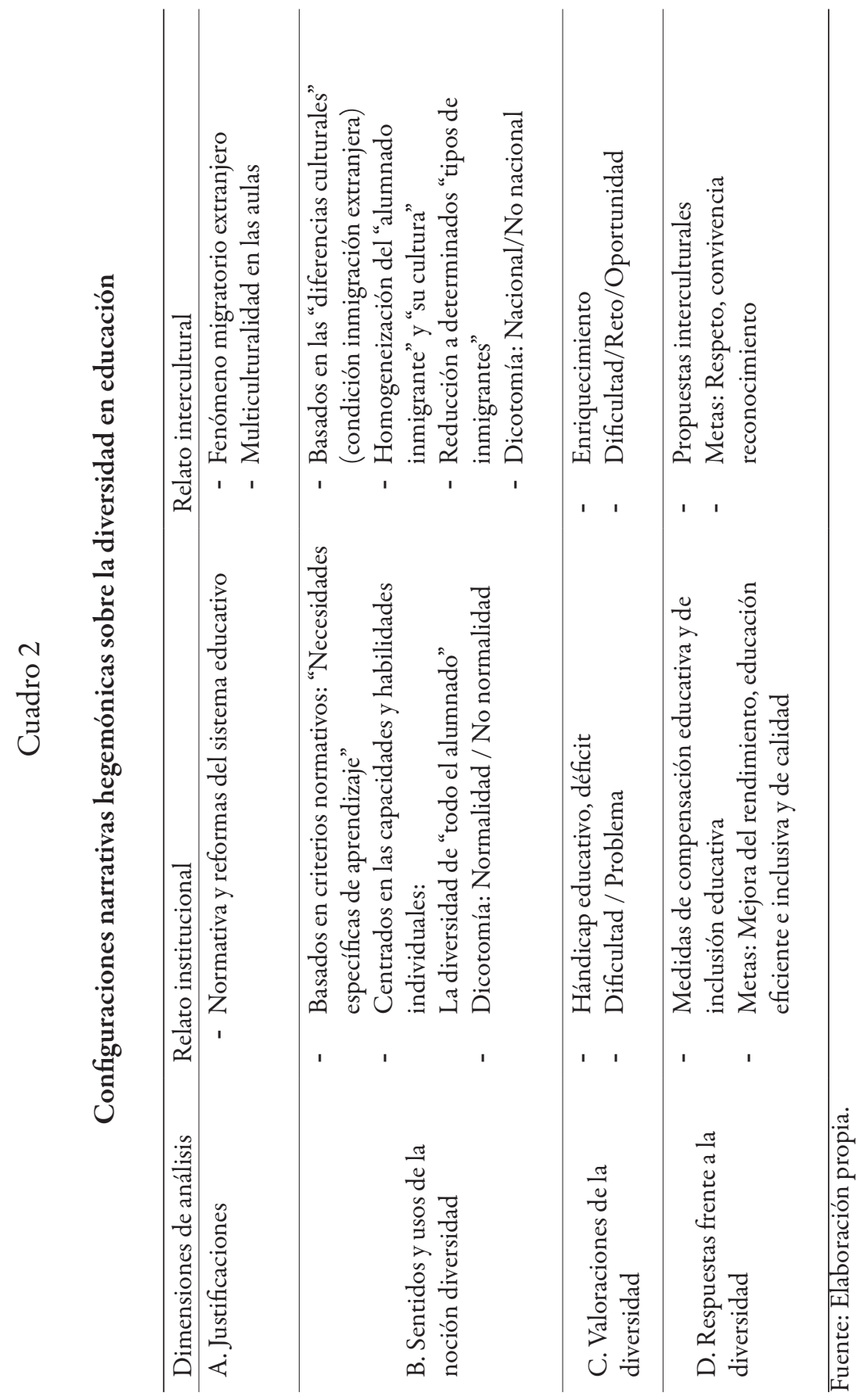


María Luisa Jiménez-Rodrigo. Doctora en Antropología Social y licenciada en Ciencias Políticas y Sociología por la Universidad de Granada. Actualmente trabaja como profesora contratada doctora del Departamento de Sociología de la Universidad de Sevilla. Sus líneas de investigación se centran en el análisis de las desigualdades de género en salud, género y participación social, y políticas sociolaborales y educativas. Publicaciones recientes: "Ir a la Universidad después de los 30: dificultades y factores facilitadores”, en Aula Abierta, vol. 42, núm. 1, España: Universidad de Oviedo (2014); "Mujeres rurales y participación social: análisis del asociacionismo femenino en la provincia de Granada (España)", en Cuadernos de Desarrollo Rural, vol. 10, núm. 72, Colombia: Pontificia Universidad Javeriana (2013); "Género y usos de drogas: dimensiones de análisis e intersección con otros ejes de desigualdad", en Oñati Socio-Legal Series, vol. 2, núm. 6, España: Instituto Internacional de Sociología Jurídica (2012).

Raquel Guzmán-Ordaz. Doctora en Sociología por la Universidad de Sevilla y Licenciada en Comunicación Social por la Universidad Autónoma Metropolitana de México. Profesora de la Universidad de Salamanca, Escuela Universitaria de Relaciones Laborales de Zamora. Líneas de investigación: desigualdades sociales desde el marco interseccional en el ámbito de las migraciones internacionales, ciudadanía, género y la educación. Publicaciones recientes: "El paradigma interseccional: rutas teóricas-metodológicas para el análisis de las desigualdades sociales", en Saletti-Cuesta, Lorena [coord.], Traslaciones en los estudios feministas, Málaga: Perséfone, Universidad de Málaga (2015); "Repensando la ciudadanía. Desafíos de la inmigración ecuatoriana en Sevilla”, en Mangliano, María José [ed.], Las mujeres latinoamericanas y sus migraciones, Argentina: Editorial Universitaria Villa María, Instituto Interdisciplinario de Estudios de Género, Universidad de Buenos Aires (2014); Márquez Lepe, Esther y García-Cano, María [coords.], Educación intercultural y comunidades de aprendizaje. Alianzas, compromisos y resistencias en el escenario andaluz, España: Catarata (2012).

Recepción: 22 de octubre de 2014.

Aceptación: 11 de febrero de 2016. 
